\title{
Measurement scale development for mobility-related quality of life among older Malaysian drivers
}

\begin{abstract}
Quality of Life (QoL) is frequently understood as subjective well-being at both individual and population levels and specific to a country or context. For individuals, mobility afforded by transport is an important predictor of QoL. This paper aims to provide an overview of theoretical approaches, particularly the selection, optimization, compensation (SOC) model, the scales used to measure quality of life, and the scale development of Mobility-related Quality of Life (M-QoL) among older drivers. A pool of items was developed from three sources: i) literature review, ii) Instrumental Activities of Daily Living (IADL) scale and iii) focus group discussion with older drivers. A survey amongst 498 older Malaysian drivers was administered to confirm internal consistency of the scale. Exploratory factor analysis revealed that the developed M-QoL scale consists of 12 items with good internal consistency $(\alpha=0.90)$, and consists of two subfactors addressing functions of driving and accessibility for independent living. Implications of the findings are discussed, including encouraging continuation of driving for those who are able to drive safely, and provision and use of alternatives to driving to help them achieve maximum mobility. Suggestions for future research are also recommended.
\end{abstract}

Keyword: Older drivers; Mobility; Quality of life; Scale development 